sein für Nachhaltigkeitszusammenhänge wird gefördert, indem ökologische und soziale Bezüge während des SBS Prozesses hinterfragt und integriert werden. Dabei hat sich die gewählte Vorgehensweise als eindeutig mittelstandstauglich bewiesen. Werden im Rahmen der Strategieentwicklung allerdings keine Entscheidungen zu Art und Umfang unternehmerischer Nachhaltigkeit getroffen, kann das Instrument SBS dieses Defizit nicht heilen.

\section{- Fazit: Kontextfaktoren stärken!}

Bei der Verankerung des Konzeptes auf Unternehmensebene stehen Eigentïmer und Geschäftsfiuhrer von kleinen und mittleren Unternehmen vor zwei Herausforderungen: Zum einen sollen sie einen konstruktiven Beitrag zur praktischen Umsetzung des politisch verwirrend formulierten Nachhaltigkeitsanspruchs leisten. Zum anderen erschwert die zunehmende Dynamisierung und Komplexität von Umfeldbedingungen die Verfolgung von Nachhaltigkeitszielen. Mittel- und langfristige Entwicklungen lassen sich aber umso besser verfolgen je klarer und stabiler die Rahmenbedingungen des Handelns sind. Daher ist es erforderlich, ein Anreizsystem zur Förderung unternehmerischer Nachhaltigkeit zu schaffen. Der auf politischer Ebene zu erzielende Grundkonsens umfasst

- Messbare Nachhaltigkeitsziele für Deutschland und die Europäische Union.

- Stabile Rahmenbedingungen der staatlichen und sozialen Subsysteme.

- Koordinierte und verständliche staatliche Förderpolitik, die über mehrere staatliche Haushaltsjahre verlässlich betrieben wird.

- Ein übersichtliches und auf Nachhaltigkeit bezogenes Umweltrecht.

Klarheit und Zuverlässigkeit bei den angestrebten politischen Zielen würde unmittelbar die Nachhaltigkeitsbestrebungen der Unternehmen befördern und Freiräume für unternehmerisches Handeln ermöglichen. Solange das nicht der Fall ist, reduziert sich die Einbeziehung von Nachhaltigkeitsaspekten auf die Frage persönlicher Werthaltungen des Unternehmers. Instrumente zur Realisierung einer nachhaltigen Unternehmensführung sind hinreichend vorhanden. Nun gilt es, die Rahmensetzungen für mehr Nachhaltigkeit zu verstärken.

\section{Anmerkungen}

(1) Vgl. Gminder, C.U. et al.: Nachhaltig managen mit der Balanced Scorecard. Erfahrungen aus einem Praxisprojekt. In: Ökologisches Wirtschaften Nr. 6/2002, S. 27-28.

(2) Informationen zum Projekt und den beteiligten Projektpartnern im Web unter www.rkw-hessen.de; die Autorinnen haben darin einzelbetriebliche Beratungen bei den Pilotunternehmen durchführt und eine Gruppe von 15 Unternehmen in einem Transferkreis fachlich betreut.

\section{Die Autorinnen}

Christel Geiger ist als Unternehmensberaterin tätig. Kontakt: Controlling Service Geiger, Friedberg , E-Mail: Geiger@c-s-geiger.de

Silke Hermann ist als Unternehmensberaterin tätig. Kontakt: Systemische Managementberatung Silke Hermann, Nussbaumstr. 8a, 65719 Hofheim a.T, Tel. 06192-952815,

E-Mail: Hermann@sysmanagement.de

\title{
Aller Anfang ist schwer
}

\section{Tourismus ist einer der dynamischsten und ökonomisch bedeutendsten globalen Wirtschaftszweige. Auch der Ökotourismus wächst - allerdings von einem klei- nen Niveau aus. Für Schritte aus der Nische bedarf es überzeugender Konzepte, die vorhandenen theoretischen Modelle auch erfolgreich in die Praxis umzuset- zen. Dies wird am Beispiel Lateinamerika illustriert.}

$\mathrm{T}$ Von Sonja Bartelt ourismus ist für viele Länder ein Hoffnungsträger der wirtschaftlichen Entwicklung. Er ist eine wichtige Devisenquelle und schafft Arbeitsplätze. Kein anderer Wirtschaftszweig hat nach Angaben der Welttourismusorganisation (WTO) von letztem Jahr Zuwachsraten von bis zu 1010 Prozent zu verzeichnen. Auf Entwicklungsländer, einschließlich der Schwellenländer, entfällt mit deutlich über 100 Milliarden US Dollar knapp ein Viertel der damit erzielten Deviseneinnahmen.

In den Zielländern führt die expansive Ausrichtung auf den Ausbau von touristischen Einrichtungen allerdings zu einer Überbeanspruchung der natürlichen Ressourcen und somit häufig zum Verlust von biologischer Vielfalt sowie der
Beeinträchtigung von sozialen und kulturellen Strukturen. Wie kaum eine andere Branche ist Tourismus jedoch auf eine intakte Natur und Umwelt angewiesen. Um einen positiven Beitrag zu einer nachhaltigen Entwicklung zu leisten, muss der Tourismus soziale, ökologische, kulturelle und wirtschaftliche Verträglichkeitskriterien erfüllen.

Trotz seiner Bedeutung wurde ihm jedoch in der internationalen Nachhaltigkeits- und Naturschutz-diskussion lange Zeit keine Beachtung geschenkt. Sowohl in der Agenda 21, der Klimarahmenkonvention als auch in der Biodiversitätskonvention wird Tourismus lediglich am Rande erwähnt.

Obwohl auch die Ökotourismus-Branche derzeit wächst, sind die Umsätze verglichen mit denen des Massentourismus eher gering. Laut Umweltprogramm der Vereinten Nationen (UNEP) liegt der Marktanteil bei drei bis fünf Prozent, was immerhin drei bis fünf Milliarden US Dollar Einnahmen aus Ökotourismus in den Entwicklungsländern bedeutet.

Durch einen langjährigen Missbrauch des Begriffs Ökotourismus wurde jedoch vielen Endverbrauchern die Sicherheit genommen, dass die Nutzung von Ökotourismuseinrichtungen zum Erhalt von Naturschutzgebieten oder der Biodiversität im Zielland beiträgt. Probleme wie die Schwierigkeit der Qualitätskontrolle oder ein fehlendes Verständnis des Begriffs Ökotourismus als solchen wurden letztes Jahr zum ersten Mal auf internationaler Ebene aufgegriffen. Die bei den Vereinten Nationen angesiedelte Kommission für Nachhaltige Entwicklung erklärte das Jahr 2002 zum internationalen Jahr des Ökotourismus.

Im Rahmen des UNEP wurden zusammen mit der WTO regionale Vorbereitungstreffen in der ganzen Welt organisiert, in denen Nichtregierungsorganisationen, staatliche Organisationen und Vertreter aus Wirtschaft und der Wissenschaft zusammenkamen, um globale Problemlagen zu diskutieren. Die Ergebnisse wurden sodann auf dem internationalen Gipfel in Quebec, Kanada im Juni 2002 zusammengetragen und verglichen (1). Ein viel versprechender Ansatz liegt in der Gestaltung des Tourismus in Naturschutzgebieten. 


\section{- Beispiel Naturschutztourismus}

Viele Nationalparks und andere Naturschutzgebiete wären heute ohne Besuchereinnahmen nicht mehr finanzierbar. Denn gerade die nicht nachhaltige Nutzung, wie intensive Landwirtschaft oder holzwirtschaftlicher Raubbau, setzt sich zumeist als kurzfristig kalkulierbares wirtschaftliches Interesse durch. Tourismuskonzepte in diesen Gebieten versuchen, wirtschaftliche und ökologische Aspekte zu verbinden, und dadurch insbesondere ökonomische Vorteile zu erzielen.

Am ehesten Erfolg versprechend sind Ansätze, die Ressourcenschutz und lokale Beteiligung mit touristischer Professionalität, unternehmerischem Denken und Marktorientierung verbinden. Genau dort setzt die seit 1999 gegründete Organisation "Mesoamerican Ecotourism Alliance" (MEA) an. Sie fördert eine Form des Tourismus, die zur Finanzierung von Schutzvorhaben beiträgt sowie einen ökonomischen Anreiz zum Erhalt von Naturräumen und ihrer Biodiversität liefert (2). Dieses Vorhaben stellt ein weltweit einmaliges Projekt dar, durch das mehrere regionale Ökotourismusprojekte in Mittelamerika versuchen, ihren internationalen Marktwert zu erhöhen. Angefangen hat es als ein Pilotprojekt der internationalen Nichtregierungsorganisation RARE Center for tropical Conservation.

\section{- Ein modellhaftes Vernetzungsprojekt}

Wichtigster Ansatz des Projekts ist es, ökologische und wirtschaftliche Denkweisen miteinander zu verbinden und den Ökotourismus wie ein Geschäft zu sehen; ein Geschäft für den Naturschutz. Das Hauptziel der MEA ist daher, die Wirtschaftskenntnisse und -expertise der teilnehmenden Parkmanager und Naturschutzorganisationen so weit zu fördern, dass sie sich zu gleichwertigen Partnern von internationalen Tourismuskonzernen etablieren und als Allianz am internationalen Markt teilnehmen können.

Die gewählte Organisationsstruktur setzt sich aus einem Vorstand, den Mitgliedern und unabhängigen Kommissionen zusammen. Die Mitglieder sind allerdings keine Einzelpersonen, sondern zehn regionale gemeinnützige $\mathrm{GmbHs}$ aus den fünf Ländern Mexiko, Guatemala, Honduras, Belize und El Salvador, die die Ökotourismusprojekte von Naturschutzgebieten in ihrem jeweiligen Land verwalten. Ein unabhängig von einer Organisation angestellter Geschäftsführer vertritt die MEA nach außen. 15 Prozent der Gewinne der von der MEA in den Zielgebieten organisierten
Reisen sollen an die Organisation zurückgeführt werden, um das Bestehen als unabhängige Institution zu ermöglichen.

\section{Sorgföltige Markteinführungsstrategie}

Zwei Jahre lang haben die Vorbereitungen gedauert, in denen die Mitglieder durch Kosten-NutzenAnalysen, Durchführbarkeitsstudien, Wirtschaftsplanung, Marketing und Kontrollverfahren ihre Tourismusprogramme soweit stabilisiert hatten, um schließlich im Jahr 2002, dem Jahr des Ökotourismus, die ersten Reisen auf dem internationalen Markt anbieten zu können. Die einmalige Qualität liegt darin, dass die Mitglieder der MEA eine große biologische und kulturelle Diversität repräsentieren: Die Reservate liegen in unterschiedlichen ökologischen Regionen, und sind entweder Biosphärenreservate oder sogar von der UNESCO ernannte Welterbestätten.

In der Produktentwicklung wurde ein Tourismusprodukt pro Reservat erarbeitet, welches über die MEA als "Marketing-Katalysator" auf dem internationalen Markt angeboten wird. Die Vorteile sind klar: Die Mitglieder nehmen nicht mehr als isolierte Kleinprojekte ohne ökonomische Breitenwirkung am internationalen Tourismusmarkt teil, sondern nehmen als eine Allianz einen größeren Stellenwert ein. So ist der jährliche Umsatz eines der Mitglieder durchschnittlich 750.000 US Dollar, zusammengenommen beträgt der Umsatz der Allianz allerdings mehrere Millionen Dollar.

\section{- Kontrolle ist notwendig}

Die Notwendigkeit zur Qualitätskontrolle der Ökotourismusprojekte ist durch die Fragilität der Gebiete besonders groß. Um MEA anzugehören, müssen sich die Mitglieder jedes Jahr rigorosen Audits unterziehen und nachweisen, dass die Erträge aus den Tourismusaktivitäten wieder direkt zu Naturschutzprojekten im eigenen Gebiet zurückgeleitet werden. Außerdem werden alle Reisen unter strikten Leitlinien ausgefïhrt, damit die kulturellen und natïlichen Ressourcen der Reservate nicht beeinträchtigt werden. Darüber hinaus kann die MEA sogar Standards für die Produktqualität setzen, indem sie internationalen Anbietern und Konsumenten gewährleistet, dass die Qualität der angebotenen Reisen überdurchschnittlich hoch ist. So müssen sich sowohl die Mitglieder wie auch die genutzte Infrastruktur einem Zertifizierungsprozess unterwerfen. Ziel dieser Qualitätskontrollen ist es, das Vertrauen der Verbraucher im Hinblick auf die Qualität von Ökotourismusprojekten wieder herzustellen.

\section{Zukunftsaussichten}

Auch die MEA musste feststellen, dass Tourismus eine Aktivität ist, die besonders stark den Regeln eines globalisierten und zunehmend dynamischen Marktes unterworfen ist - mit entsprechenden Konsequenzen für die Komplexität der Planung und der Vielfalt der beteiligten Akteure. Durch den Einbruch auf dem internationalen Tourismusmarkt, vor allem nach den Terroranschlägen vom 11. September 2001 in New York, ist die erfolgreiche Durchführung des Projekts zu einer Herausforderung geworden. Erschwerend kommt hinzu, dass die Reisen durch ihre Eigenschaften und einen relativ hohen Preis nur auf ein kleines Marktsegment zugeschnitten sind.

Von den zehn geplanten Reisen in 2002 wurden nur fünf durchgeführt, und ein Jahr nach Entstehen des Projekts wird daher ein Großteil der Gewinne noch für die Administrationen der einzelnen Institutionen aufgewendet. Positiv ist, überhaupt eine Struktur geschaffen zu haben, die die Durchfuihrung dieser Projekte ermöglicht, der Bevölkerung Einnahmen verschafft und den sowieso bestehenden Tourismus in den Gebieten reguliert. In der Zukunft sollen allerdings konkretere Projekte gefördert werden, für die die Naturschutzgebiete momentan keine Gelder haben. Beispiele sind nötige Weiterbildungsmaßnahmen für die ansässige Bevölkerung über die Bedeutung des Schutzes ihrer natürlichen Ressourcen sowie Forschungsprojekte über nur in diesen Gebieten vorkommende Arten. Man hofft, dass dieses Modell anderen Regionen als Anreiz dienen kann, um Tourismus erfolgreich als Finanzierungsquelle für Naturschutzprojekte in Schwellenländern einzusetzen, ohne die natürlichen Ressourcen zu vermindern.

\section{Anmerkungen}

(1) Weitere Informationen auf www.uneptie.org/pc/ tourism/ecotourism/iye.htm

(2) Nähere Informationen auf www.ecotourismalliance.com

\section{Die Autorin}

Sonja Bartelt hat mehrere Jahre das Ökotourismusprogramm eines der Mitglieder der MEA geleitet und betreut zurzeit die Geschäftsstelle der Vereinigung für ökologische Wirtschaftsforschung (VÖW). Kontakt: VÖW-Geschäftsstelle, Potsdamer Str. 105, 10785 Berlin. Tel. 030-8851800, E-Mail: sonja.bartelt@gmx.de 
(c) 20I0 Authors; licensee IÖW and oekom verlag. This is an article distributed under the terms of the Creative Commons Attribution Non-Commercial No Derivates License (http://creativecommons.org/licenses/by-nc-nd/3.o/), which permits unrestricted use, distribution, and reproduction in any medium, provided the original work is properly cited. 